\title{
Resistance Exercise May Improve Spatial Awareness and Visual Reaction in Older Adults
}

\author{
Maren S. Fragala, Kyle S. Beyer, Adam R. Jajtner, Jeremy R. Townsend, Gabriel J. Pruna, \\ Carleigh H. Boone, Johnathan D. Bohner, David H. Fukuda, Jeffrey R. Stout, and \\ JAY R. Hoffman \\ Institute of Exercise Physiology and Wellness, University of Central Florida, Orlando, Florida
}

\begin{abstract}
Fragala, MS, Beyer, KS, Jajtner, AR, Townsend, JR, Pruna, GJ, Boone, CH, Bohner, JD, Fukuda, DH, Stout, JR, and Hoffman, JR. Resistance exercise may improve spatial awareness and visual reaction in older adults. J Strength Cond Res 28(8): 2079-2087, 2014-Aerobic exercise has been shown to counteract age-related neurological decrements that are associated with cognitive and physical impairments. However, the effects of resistance exercise on cognition, reaction, and neurotrophins are largely unknown. We examined changes in spatial awareness, visual and motor reaction, and circulating brain-derived neurotrophic factor (BDNF) in response to a resistance exercise intervention in older adults (aged $70.6 \pm 6.1$ years). Spatial awareness was evaluated before and after training with a Neurotracker perceptual 3-dimensional objecttracking device. Peripheral, visual, motor, and physical reaction times were evaluated using a Dynavision visuomotor device. Circulating BDNF was assayed. Data were analyzed for clinical significance using magnitude-based inferences calculated from independent $t$-tests. Clinical interpretations of the analyses revealed that resistance exercise training was "likely beneficial" for improving spatial awareness and visual and physical reaction times. Each improved by 40.0, 14.6, and 14.0\%, respectively. Circulating BDNF and motor reaction time displayed no apparent meaningful changes. Thus, resistance exercise training may be an effective means to preserve or improve spatial awareness and reaction with aging.
\end{abstract}

KEY WoRDS aging, strength training, cognitive function, seniors, BDNF, neurotrophins

\section{INTRODUCTION}

W

ith aging, neurological changes occur in the brain and periphery resulting in cognitive declines and physical ailment. In the aging brain, neurons lose size, myelination, and the ability

Address correspondence to Dr. Maren S. Fragala, maren.fragala@ucf.edu. 28(8)/2079-2087

Journal of Strength and Conditioning Research (C) 2014 National Strength and Conditioning Association to regenerate (32). In the periphery, motor units undergo dysregulated synchronization, discharge, and conduction velocity with aging (11). Cognitively, neurological changes can alter central processing and increase the risk of developing symptoms of dementia (33). Physically, neuromuscular changes can lead to fatigue-induced motor incoordination and result in physical disability, accidents, falls, and skeletal injuries (21). When combined, central and peripheral neurological changes can impair the ability of older adults to perceive and respond to everyday stimuli leading to consequential impairments in quality of life.

Spatial awareness is a component of cognitive function that allows an individual to perceive and understand the temporal and spatial components of the surrounding environment (13). With normal aging, the speed at which information can be processed slows down (39) and spatial awareness declines (5). Deficits in visual-spatial functioning are predictive of further cognitive decline (20). In addition, the ability to react to stimuli slows down with aging (19) because of both sensory and motor changes $(17,36)$. Consequently, reaction time substantially slows down in the early stages of dementia (35) and has been shown to identify older adults at the risk of developing dementia (18). Both the ability to perceive the surrounding environment and the ability to react to stimuli are paramount to accident avoidance and safety in aging adults.

Although, mental stimuli in various interventions have typically been recommended for cognitive maintenance, some studies have implicated the role of physical exercise in reducing the cognitive deterioration in older adults $(2,9,42)$. To date, most exercise interventional studies have implemented aerobic type exercise, where cognitive benefits were observed $(2,42,48)$ especially in tasks requiring executive control (9). Few studies to date have evaluated the effects of resistance-type exercise on cognitive function and reaction in older adults. Resistance exercise training has been shown to be a safe and effective method for conditioning the peripheral neuromuscular system and improving muscle strength and power in older adults (1). However, its cognitive effects on spatial awareness and subsets of visual and motor reaction are largely unknown. In contrast to repetitive aerobic-type exercise, resistance-type exercise requires 
judgment of proprioception and evaluation of loads, which activate different regions of the brain (6,8). Similarly, although aerobic exercise results in primarily cardiovascular and circulatory adaptations (26), resistance-type exercise has been shown to result in profound neurological adaptations within the first several weeks of training (38).

The mechanisms underlying the neurological adaptations to exercise intervention are yet to be elucidated, but likely involve circulating growth factors and neurotrophins (29), a family of proteins that are essential for the development, differentiation, and survival of neurons (12). Of the neurotrophins, brainderived neurotrophic factor (BDNF) is the most prevalent neurotrophin in the central nervous system where it plays several pivotal roles in synaptic plasticity and neuronal survival (45). Moreover, BDNF plays a major role in regulating axonal and dendritic growth and guidance, long-term potentiation, and participation in neurotransmitter release (4). Previous studies have shown that BDNF measured in the serum is associated with cardiopulmonary fitness, psychomotor processing speed, cognition (44), hippocampal size (14), and physical activity (16). However, it is not clear whether resistance exercise training affects circulating BDNF in older adults.

A few seminal studies have shown some cognitive improvements from resistance training in older adults $(30,31)$. Nevertheless, to our knowledge, no prior studies have evaluated the effects of resistance exercise training on spatial awareness, visual and motor reaction time in older adults. Additionally, to our knowledge, few studies have evaluated the underlying mechanisms of such changes and whether BDNF is related to cognitive changes resulting from resistance training in older adults. We are unaware of any studies that have evaluated serum BDNF, a biomarker associated with remodeling of the neuromuscular junction, in response to an intervention designed to stimulate and train a large motor unit pool over several weeks. Thus, the overarching objective of this study was to evaluate specific changes in spatial awareness and reaction to short-term resistance exercise training in previously untrained older adults. We also sought to determine if the circulating levels of BDNF change in response to a resistance training intervention in older adults.

\section{Methods}

\section{Experimental Approach to the Problem}

Spatial awareness, visual and motor reaction, and circulating BDNF were evaluated at baseline and after 6 weeks of resistance training or no intervention (control) in older adults by using a randomized controlled design. Spatial awareness was evaluated before and after training with a Neurotracker perceptual 3-dimensional (3D) objecttracking device. Peripheral, visual, motor, and physical reaction times were evaluated using a Dynavision visuomotor device. Circulating BDNF was assayed using Luminex MagPix technologies. Differences between resistance exercise training and control groups were analyzed for clinical significance using magnitude-based inferences calculated from independent $t$-tests.

\section{Subjects}

Twenty-five healthy older adults (age $>60$ years) volunteered to participate in the study (age $70.64 \pm 6.11$ years; height $1.69 \pm 0.09 \mathrm{~m}$; weight $80.72 \pm 19.42 \mathrm{~kg}$ ) and were randomized into the training $(n=13)$ or control group $(n=12)$. All the participants were free from cardiovascular, pulmonary, or metabolic symptoms and disease and were free from musculoskeletal injuries or recent surgeries. The participants were informed of study procedures and provided an informed written consent before participating in any of the study procedures. The study was approved by the Institutional Review Board at the University of Central Florida.

\section{Procedures}

All the participants completed all tests before and after 6 weeks of resistance training or control. All tests were completed on the same day of testing in a standardized order. Compliance to the resistance exercise intervention was $100 \%$.

\section{Spatial Awareness}

Spatial awareness was measured as a threshold score on the Neurotracker (CogniSens Athletic Inc., Montreal, Canada) 3D multiple object-tracking device at baseline and after the intervention or control period (Figure 1). Threshold scores were derived based on object movement speeds in centimeters per second as previously described (28). During the test, the participants wore $3 \mathrm{D}$ glasses and sat in the 3D simulator cave. Each testing session consisted of 20 trials in which the participants were required to track designated objects as they moved through space. The speed of object motion was adjusted for subsequent trials based on prior scoring until a threshold was determined. The score was considered to be the threshold for effective perception and processing of visual information sources (15). Test-retest reliability for the Neurotracker threshold values was determined using 12 healthy older men and women (age $>60$ years) measured 7 weeks apart. The intraclass correlation coefficient (ICC) was 0.77 , and standard error of measurement $(S E M)$ was 0.25 . In addition, there was no significant difference between the mean Neurotracker threshold values from the reliability testing (mean threshold $\pm S E M$; Pre: $0.73 \pm 0.11$ vs. Post: $0.75 \pm 0.10$ ).

\section{Reaction Time}

All reaction time measurements were assessed on the Dynavision D2 Visuomotor Training Device (D2; Dynavision International LLC, Chester, OH, USA), which features a $4^{\prime \prime} \times 4^{\prime \prime}$ display containing 64 individual lighted buttons (Figure 2). Peripheral visuomotor reaction time was determined from a 60 -second trial in which the participants were instructed to tap buttons as fast as possible on the display as they illuminated in a randomized pattern. Average reaction time was calculated and used in subsequent analysis. The visual, motor, and physical reaction times were assessed from 10 trials in which the participants were instructed to

2080 Journal of Strength and Conditioning Research 


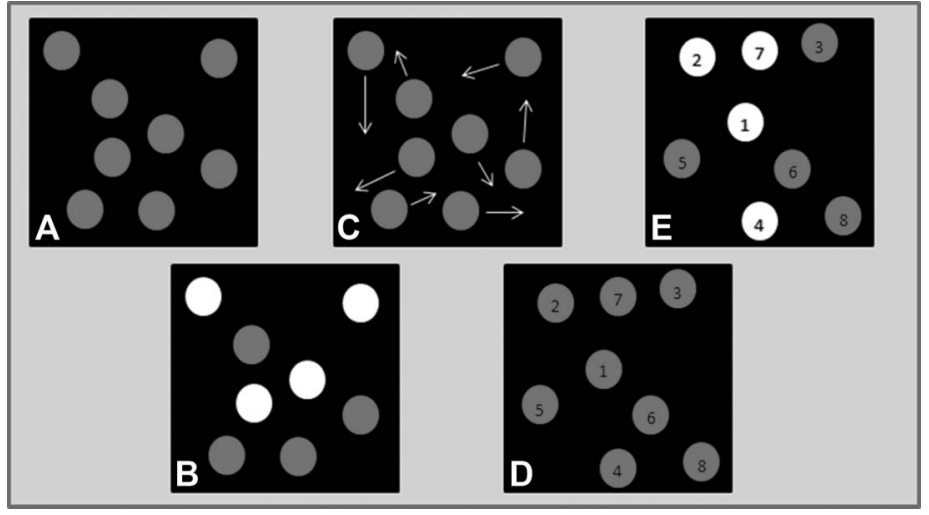

Figure 1. Diagram of the Neurotracker 3D perceptual tracking test. The test was conducted in a threedimensional hood where the participants tracked 4 selected objects as they moved at varying speeds through space. Scoring was based on the threshold speed for accurate perception and processing. A) Starting screen. B) Four of the objects were illuminated. C) Objects return to baseline color and move in space for 8 seconds. D) Objects stop motion and are numbered. E) The participants select the objects that were illuminated in step B.

starting point. Motor reaction time was determined by the length of time it took to hit the light after lifting the hand from the starting point. Physical reaction time was calculated as the combination of the motor and visual reaction times. The reliability $(\mathrm{ICC}=$ 0.88 ) of the Dynavision tests has been previously reported (25) on a group of 34 subjects.

\section{Blood Sample Collection and Preparation}

Resting blood samples were collected in the morning after an overnight fast. Blood was drawn from a forearm vein into serum separator tubes for serum sample preparation. Serum tubes were allowed to clot for $30 \mathrm{mi}-$

hit an illuminated button and return to the original position with the same hand as fast as possible. Visual reaction time was calculated as the time that elapsed before each participant responded to the light by lifting his/her hand from the

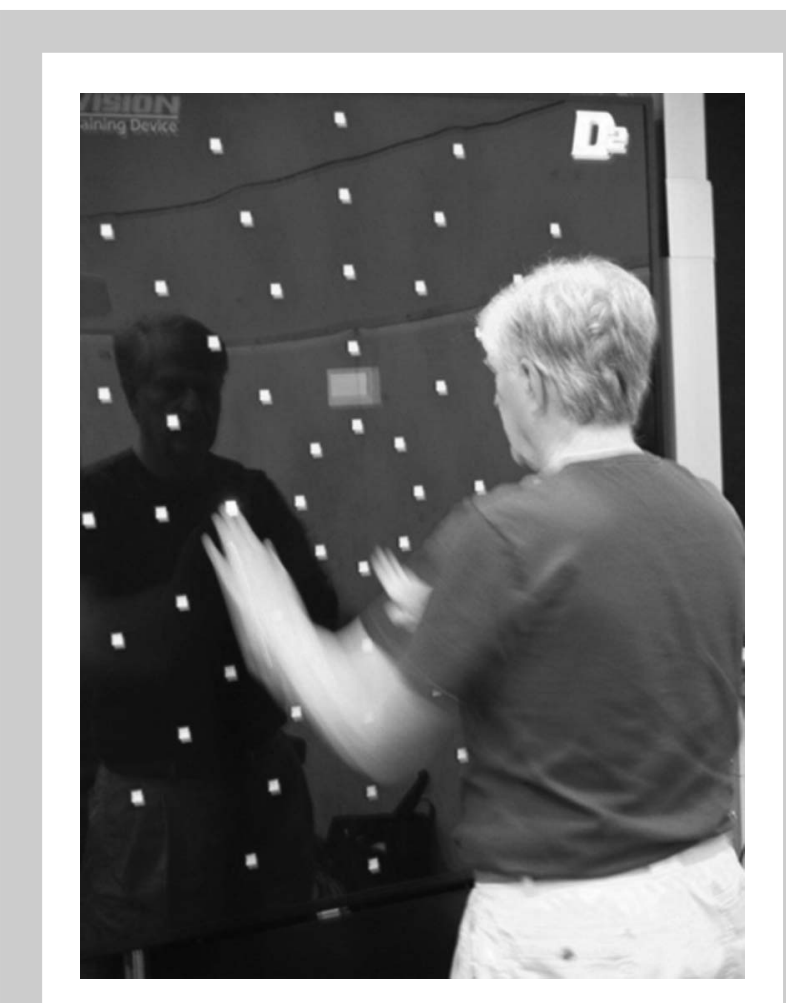

Figure 2. The Dynavision D2 visuomotor device was used to quantify peripheral visuomotor, visual, motor, and physical reaction times through a series of tests. nutes before centrifugation. Serum samples were then separated by centrifugation for 10 minutes at $1,000 g$ at $-4^{\circ} \mathrm{C}$. The serum was immediately aliquoted into designated preservative tubes and stored as samples at $-80^{\circ} \mathrm{C}$ until analysis. Before analysis, the samples were thawed only once, centrifuged to remove particulates, and mixed completely by vortexing.

\section{Serum Brain-Derived Neurotrophic Factor}

Serum BDNF was measured by a commercially available immunoassay kit (Milliplex MAP Catalog No: HNDG3MAG36K, Billerica, MA, USA). Before analysis, the serum samples were diluted in the ratio of 1:100 in an assay buffer. Twentyfive microliters of the diluted serum sample was added into the appropriate microtiter plate wells containing $25 \mu \mathrm{l}$ of matrix solution. Twenty-five microliters of fluorescentcoded magnetic antihuman BDNF microspheres was then added to each well, and the samples were incubated overnight with shaking at $4^{\circ} \mathrm{C}$. Well contents were removed, and after washing, $25 \mu \mathrm{l}$ of detection antibody was added to each well. After a 1 -hour incubation at room temperature, $25 \mu$ l of streptavidin-phycoerythrin was added to each well. After a 30-minute incubation, the contents of the wells were removed, and $100 \mu \mathrm{l}$ of sheath fluid was added to each well. Fluorescence was measured on a MAGPIX instrument equipped with XPONENT software (Luminex Corp.; Luminex, Austin, TX, USA). The concentration of BDNF in the samples was determined using a 5-parameter logistic curve fit. The sensitivity of this assay is $0.23 \mathrm{pg} \cdot \mathrm{ml}^{-1}$, and intraassay and interassay precisions are 5.4 and $5.3 \%$, respectively. All samples were run in duplicate and thawed only once.

\section{Training Protocol}

Before beginning the training program, the participants completed 2 days of training familiarization in which 
TABLE 1. Changes in spatial awareness (Neurotracker threshold speed), reaction, and BDNF from 6 weeks of resistance exercise training or control in older adults. ${ }^{*} \dagger$

\begin{tabular}{|c|c|c|c|c|}
\hline & \multicolumn{2}{|c|}{ Exercise $(n=13)$} & \multicolumn{2}{|c|}{ Control $(n=12)$} \\
\hline & Pre & Post & Pre & Post \\
\hline Neurotracker threshold speed (au) & $0.44 \pm 0.26$ & $0.60 \pm 0.21$ & $0.73 \pm 0.36$ & $0.75 \pm 0.35$ \\
\hline Peripheral visuomotor reaction time (s) & $1.12 \pm 0.23$ & $1.03 \pm 0.20$ & $1.14 \pm 0.19$ & $1.07 \pm 0.17$ \\
\hline Visual reaction time $(\mathrm{s})$ & $0.53 \pm 0.20$ & $0.45 \pm 0.05$ & $0.47 \pm 0.05$ & $0.46 \pm 0.03$ \\
\hline Motor reaction time $(\mathrm{s})$ & $0.36 \pm 0.10$ & $0.31 \pm 0.07$ & $0.39 \pm 0.09$ & $0.37 \pm 0.08$ \\
\hline Physical reaction time (s) & $0.88 \pm 0.24$ & $0.76 \pm 0.10$ & $0.86 \pm 0.11$ & $0.83 \pm 0.08$ \\
\hline Serum BDNF $\left(\mathrm{ng} \cdot \mathrm{ml}^{-1}\right)$ & $30.8 \pm 15.3$ & $28.6 \pm 15.0$ & $28.3 \pm 12.7$ & $27.8 \pm 11.2$ \\
\hline
\end{tabular}

*BDNF $=$ brain-derived neurotrophic factor.

$\dashv$ Data are presented as mean $\pm S D$.

exercises were taught and resistance was determined. The training group underwent 2 resistance training sessions per week for 6 weeks, with at least 48 hours between all training sessions to allow for proper recovery. Each training session began with a dynamic warm-up consisting of body weight squats, high knee walking, heel kick walking, torso rotation, and arm rotations. Full-body workouts were performed during each session with 7 or 8 of the following exercises performed each day. Exercises included leg extensions, leg curls, seated rows, lat pull-downs, modified squats, modified split squats, modified stiff-legged dead-lifts, biceps curls, chest presses, shoulder presses, tricep extensions, abdominals, and calf raises. Three sets of each exercise were performed with the exercises and the number of repetitions systematically varying on a weekly basis ranging from 8 to 15 repetitions. The load was adjusted with the number of

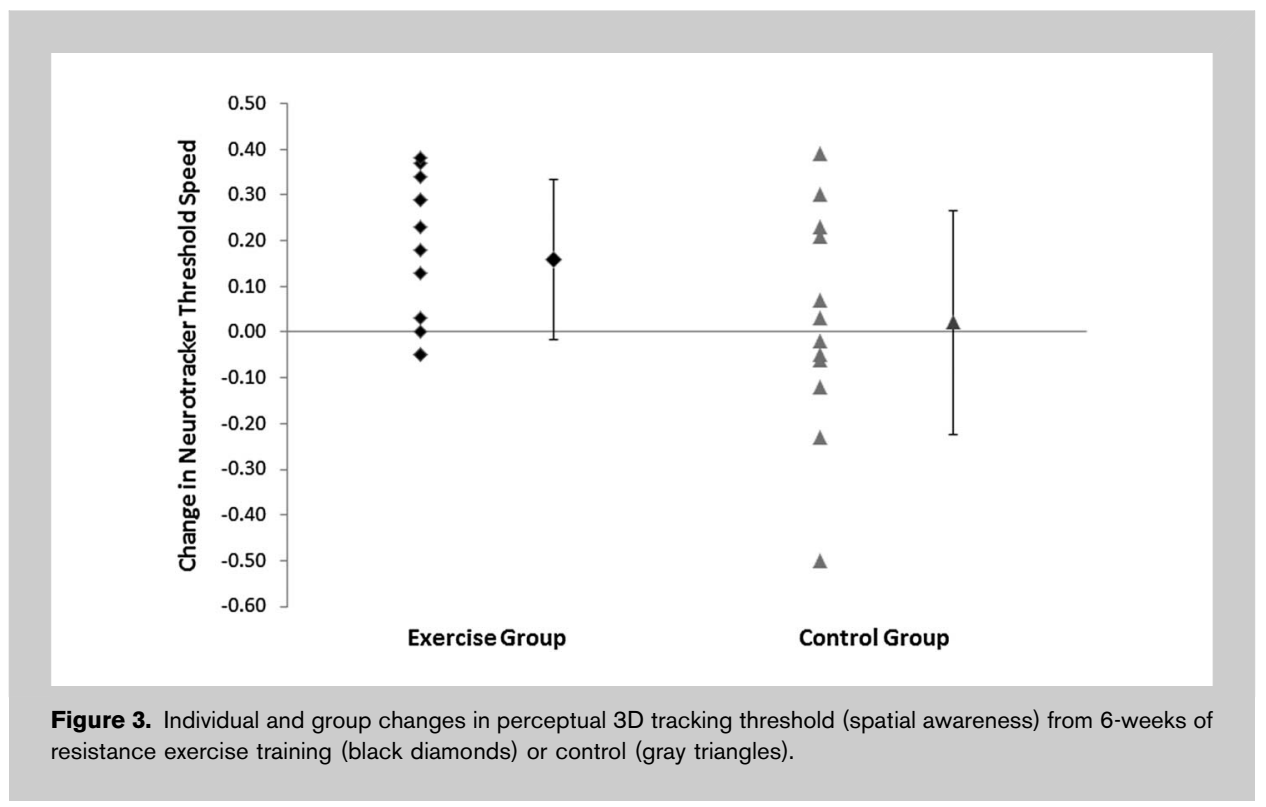

repetitions assigned. The OMNI scale (37) of perceived exertion for resistance-type exercise ranging from 0 to 10 was used to assess difficulty during the training. Load was prescribed at a "moderate" perceived exertion corresponding to a " 5 " or " 6 " on the scale. All training sessions were supervised one-on-one training sessions overseen by a Certified Strength and Conditioning Specialist. The exercise program followed the recommended guidelines for older adults by the American College of Sports Medicine and the National Strength and Conditioning Association.

\section{Statistical Analyses}

The effects of resistance exercise were calculated as the changes from pretraining to posttraining cognitive measurements among the exercise and control groups. Magnitudebased inferences were used to identify clinical differences in the cognitive changes between the exercise and control groups. Several studies have supported the use of magnitude-based inference statistics as a complementary tool for null hypothesis testing to reduce errors in interpretation $(3,23)$, and to provide more clinically meaningful results. Magnitude-based inference statistics avoid the problem of interdependence of observations by using within-subject modeling, where repeated measures are combined into a single measure (unit of change) for analysis. The precision of the magnitude of inference was set at $90 \%$ confidence limits, using a $p$ value derived from an independent $t$-test. Threshold values for 
TABLE 2. Magnitude-based clinical inferences on changes in neural function with resistance exercise training. *

\begin{tabular}{|c|c|c|c|c|c|c|}
\hline \multirow[b]{2}{*}{ Variable } & \multicolumn{2}{|c|}{ Mean change (\% change) } & \multirow{2}{*}{$\begin{array}{c}\% \\
\text { Positive }\end{array}$} & \multirow{2}{*}{$\begin{array}{c}\% \\
\text { Trivial }\end{array}$} & \multirow{2}{*}{$\begin{array}{c}\% \\
\text { Negative }\end{array}$} & \multirow{2}{*}{$\begin{array}{l}\text { Clinical } \\
\text { inference }\end{array}$} \\
\hline & Exercise & Control & & & & \\
\hline $\begin{array}{l}\text { Neurotracker threshold } \\
\text { speed (au) }\end{array}$ & $0.178 \pm 0.163(40.0)$ & $0.021 \pm 0.246(2.9)$ & 85.0 & 14.3 & 0.8 & $\begin{array}{l}\text { Likely } \\
\text { beneficial }\end{array}$ \\
\hline $\begin{array}{l}\text { Peripheral visuomotor } \\
\text { reaction time (s) }\end{array}$ & $-0.102 \pm 0.072(9.0)$ & $-0.072 \pm 0.110(6.3)$ & 38.5 & 57.7 & 3.8 & Unclear \\
\hline Visual reaction time (s) & $-0.078 \pm 0.182(14.6)$ & $-0.008 \pm 0.048(1.7)$ & 76.3 & 19.8 & 3.9 & $\begin{array}{l}\text { Likely } \\
\text { beneficial }\end{array}$ \\
\hline Motor reaction time (s) & $-0.053 \pm 0.084(14.8)$ & $-0.022 \pm 0.101(5.6)$ & 61.8 & 28.9 & 9.4 & Unclear \\
\hline Physical reaction time (s) & $-0.123 \pm 0.188(14.0)$ & $-0.031 \pm 0.112(3.6)$ & 80.2 & 17.2 & 2.6 & $\begin{array}{l}\text { Likely } \\
\text { beneficial }\end{array}$ \\
\hline Serum BDNF $\left(\mathrm{ng} \cdot \mathrm{ml}^{-1}\right)$ & $-2.26 \pm 6.4(-7.3)$ & $-0.46 \pm 5.23(-1.63)$ & 3.5 & 64.4 & 32.1 & $\begin{array}{c}\text { Very unlikely } \\
\text { beneficial }\end{array}$ \\
\hline
\end{tabular}

${ }^{*} \mathrm{BDNF}=$ brain-derived neurotrophic factor.

positive and negative effects were calculated by multiplying the standard deviations of baseline values by $20 \%(22)$. Inferences on true differences between the exercise and control groups were determined as positive, trivial, or negative according to methods that were previously described (23). Inferences were based on the confidence interval range relative to the smallest clinically meaningful effect. Unclear results are reported if the observed confidence interval overlaps both positive and negative values. The probability of the effect was evaluated according to the following scale: $<0.5 \%$, most unlikely; $0.5-5 \%$, very unlikely; $5-25 \%$, unlikely; $25-75 \%$, possibly; $75-95 \%$, likely; $95-99.5 \%$, very likely; and $>99.5 \%$, most likely (22).

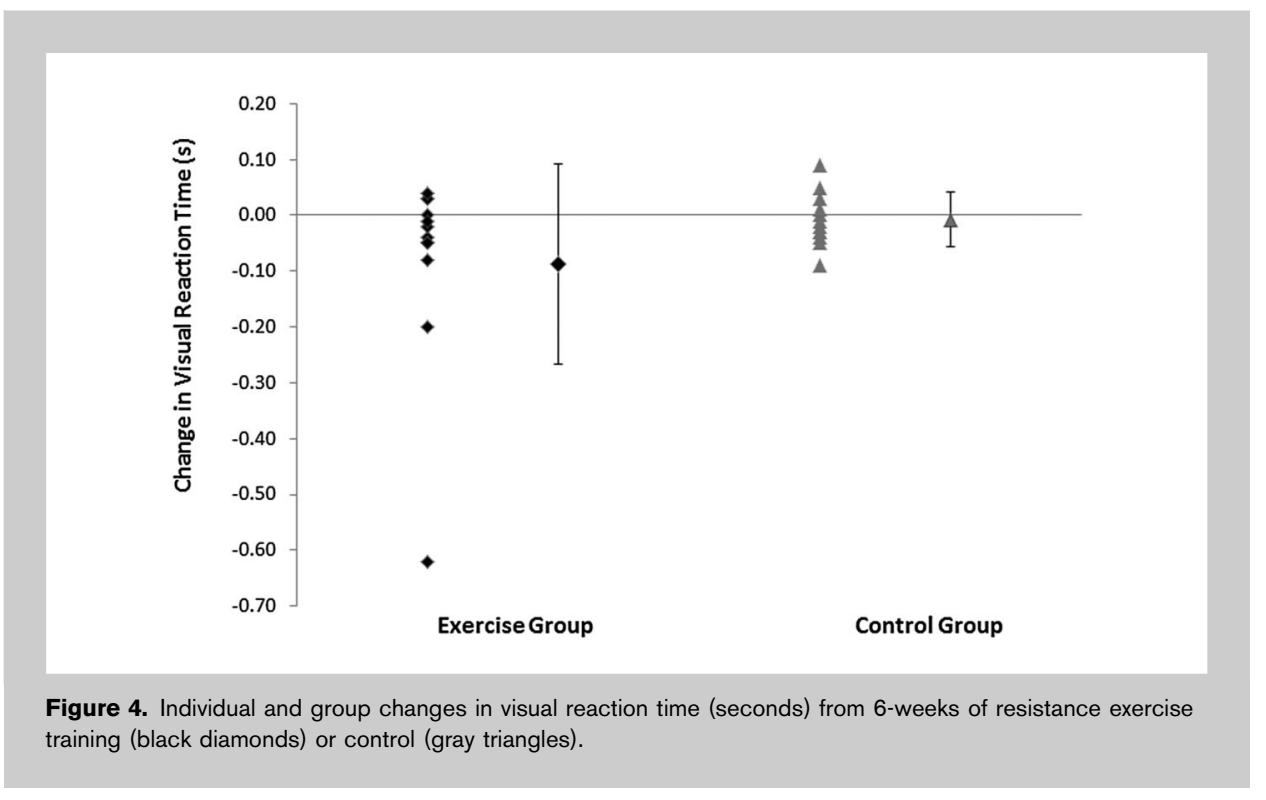

\section{Results}

Baseline Neurotracker 3D perceptual tracking threshold speed (spatial awareness) was $0.44 \pm 0.26$ and $0.73 \pm 0.36$ au for the training group and control group, respectively (Table 1). Baseline peripheral visuomotor, visual, motor, and physical reaction times for the training group were $1.138 \pm 0.228$ seconds, $0.533 \pm 0.208$ seconds, $0.359 \pm$ 0.109 seconds, and $0.877 \pm 0.248$ seconds, respectively. The control group displayed similar baseline scores for peripheral visuomotor, visual, motor, and physical reaction times, $1.141 \pm 0.192$ seconds, $0.470 \pm 0.054$ seconds, $0.390 \pm 0.094$ seconds, and $0.859 \pm 0.114$ seconds, respectively. After the 6-week intervention, the training group experienced a greater mean change than did the control group in all cognitive variables (Figure 3). The mean changes for each group and the differences between the groups can be seen in Table 2. Magnitudebased inferences revealed that resistance exercise training is likely beneficial for improving Neurotracker threshold speed, visual reaction time, and physical reaction time. The greatest difference between the 2 groups $(0.160 \pm 0.150)$ was observed in spatial awareness (Neurotracker threshold speed) where the exercise training group improved by $0.178 \pm 0.163(40.0 \%)$ and the control group improved by 


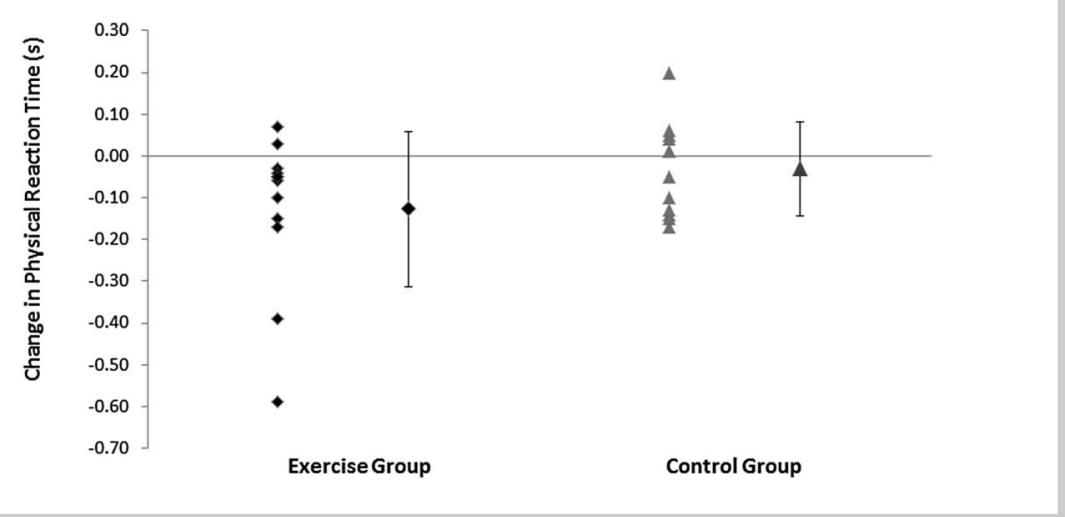

Figure 5. Individual and group changes in physical reaction time (seconds) from 6-weeks of resistance exercise training (black diamonds) or control (gray triangles).

effects of resistance training on circulating BDNF. Additionally, changes in circulating BDNF did not seem to correlate with any measure of neural function.

\section{Discussion}

Our results indicate that specific measures of cognitive function and reaction in older adults may show improvements from short-term resistance exercise of a 6-week duration. Although, previous research has shown resistance exercise to improve some aspects of cognitive function $(30,31)$, to our knowledge,

$0.021 \pm 0.246(2.9 \%)$ from the baseline (Table 2). Neurotracker threshold speed displayed an $85 \%$ probability of improving with resistance exercise training, indicating a qualitative inference of "Likely Beneficial." Similarly, the exercise training group improved in visual reaction time by $0.078 \pm 0.182$ seconds $(14.6 \%)$ and physical reaction time by $0.123 \pm 0.188$ seconds (14.0\%) (Table 2) (Figures 4 and 5). The control group changed by $0.008 \pm 0.048$ seconds $(1.7 \%)$ and $0.031 \pm 0.112$ seconds $(3.6 \%)$ for visual and physical reaction times, respectively. Visual and physical reaction times displayed 76.3 and $80.2 \%$ probabilities of improving from resistance exercise training, indicating qualitative inferences of "Likely Beneficial." Magnitude-based inferences and probabilities of beneficial, negligible, and negative effects are given in Table 2.

Confidence intervals that substantially overlapped both positive and negative values revealed "unclear" effects of resistance exercise training on peripheral visuomotor reaction time and motor reaction time. Despite being statistically unclear by magnitude-based inferences, peripheral visuomotor reaction time and motor reaction time improved by $0.102 \pm 0.072$ (9.0\%) seconds and $0.053 \pm 0.084$ (14.8\%) seconds, respectively, in the resistance exercise training group. Possibly beneficial effects of 38.5 and $61.8 \%$ for peripheral visuomotor reaction time and motor reaction time, respectively, were observed. The probabilities of negligible or trivial effects of resistance training were 57.7 and $28.9 \%$ for peripheral visuomotor reaction time and motor reaction time, respectively.

Baseline BDNF was significantly lower in women than in men $\left(22.4 \pm 14.2\right.$ vs. $\left.35.5 \pm 12.8 \mathrm{ng} \cdot \mathrm{ml}^{-1}\right)$, but training and control groups, consisting of both men and women, did not differ. Although circulating BDNF tended to decrease in the training group ( $-7.3 \%$ mean change) after 6 weeks, no significant or clinically meaningful changes were observed. With $64.4 \%$ probability of the changes in BDNF being trivial, clinical inferences indicated "very unlikely beneficial" prior studies have not evaluated the effects of resistance exercise training on spatial awareness and distinct visual and motor aspects of reaction time. Moreover, to our knowledge, no prior studies have evaluated the effects of only 6 weeks of resistance exercise training on any cognitive measures in older adults.

Spatial awareness exhibited an $85 \%$ positive probability of improving from resistance exercise training. Spatial awareness involves higher order processing of the temporal and spatial components of the surrounding environment (13) and reflects the function of the superior temporal cortex (24). The decline in spatial awareness (5) and slowing down of the speed at which information can be processed (40) with aging can make tasks such as driving dangerous for older adults. Although spatial awareness is vital to interacting with dynamic situations in the environment, quantifying spatial awareness has previously been challenged by the lack of a feasible and accessible testing apparatus. One prior study has shown that the use of 3D virtual environments can improve spatial awareness in children with physical disabilities (49). Similarly, although studies in older adults are lacking, a recent study reported lower baseline threshold neurotracking speeds in older adults than in younger adults (28).

Interventions that can improve spatial awareness are vital to healthy aging and preserving cognitive functioning in dementia. Our data show that resistance exercise training may be an important means to improve spatial awareness in older adults. Mechanisms as to how resistance exercise may improve spatial awareness are not known. However, prior research has shown that physical exercise can prevent agerelated atrophy in the temporal lobe of older adults (7), and some research has uncovered the potentially mediating role of circulating growth factors and neurotrophins in neural adaptations (29). Moreover, physical exercise has been shown to affect the structure and function of the brain via 
increased cerebral blood flow; improved transport; and use of nutrients, neurogenesis, and neurotransmission $(46,47)$.

Physical reaction time, which was calculated as the combined measure of visual and motor reaction times, displayed an $80.2 \%$ probability of improving with exercise. Similarly, previous research has shown a positive relationship between high levels of physical activity and faster reaction times (42). This improved ability to respond to a stimulus seems to be related to an improvement in visual reaction time rather than to motor reaction time. Visual reaction time showed a $76.3 \%$ probability of improving with resistance exercise training, whereas motor reaction time had only a $61.8 \%$ probability of improving with exercise. Visual reaction time was defined in this study as the amount of time to recognize a light stimulus and release a button. Previous research has shown that faster reaction times are related to faster brain wave frequencies (43). Hence, although not measured in this study, it is possible that resistance exercise training has a profound influence on brain wave activity. Prior research has reported that engaging in $>12$ hours of intense exercise a week correlated with an increased frequency in brain waves (27).

Peripheral visuomotor and motor reaction times revealed unclear effects of resistance exercise training. Unclear findings from magnitude-based inferences indicate that the probabilities of a positive or negative outcome are both substantial. Previous research has suggested that when unclear results are obtained, further research may be necessary in a larger sample size to decipher likely results (3). Nevertheless, peripheral visuomotor and motor reaction times did demonstrate substantial probabilities of improving with resistance exercise, 38.5 and $61.8 \%$, respectively. Previous research has indicated that such probabilities could be interpreted as "possibly beneficial" (23). We elected to conservatively report that these results were "unclear." The reason that peripheral visuomotor and motor reaction times did not seem to benefit from resistance exercise training is not well understood. It could be that our small sample size and relatively high degree of variance for each measure contributed to the results. However, prior research has shown that motor reaction time does improve with physical activity (10). It may be that our "unclear" effect of resistance training on peripheral visuomotor and motor reaction times is valid for older adults. Prior research has shown that motor responses to resistance training are attenuated in older adults compared with that in younger adults (34). Perhaps, although visual reaction time improves with exercise training in older adults, the motor aspect is less adaptable with resistance exercise training. Further research is needed to provide a more definitive determination of the impact of resistance exercise training on peripheral visuomotor and motor reaction times.

Surprisingly, circulating BDNF did not show statistically or clinically meaningful changes with resistance exercise training. As previous studies have shown that BDNF measured in the serum is associated with cardiopulmonary fitness, psychomotor processing speed, cognition (44), hippocampal size (14), and physical activity (16), we had expected to see increases in circulating BDNF with resistance training that paralleled neural functioning improvements. The reason for the lack of observed changes in resting circulating BDNF may be attributable to the time frame of measurement. We measured fasting and resting concentrations of circulating BDNF before and after the 6-week intervention to assess potential chronic changes. However, recent evidence in younger adults has suggested that circulating changes may be more transient, and last only 20 minutes after exercise and are dependent on exercise intensity (41). Thus, although we could not attribute our observed neural functional improvements to measured changes in circulating BDNF, it is possible that BDNF still was involved in the neural adaptations in the acute time frame surrounding each bout of exercise. Because BDNF plays a major role in regulating axonal and dendritic growth and guidance, long-term potentiation, and participation in neurotransmitter release (16), its elevations in serum may be more transient. Further, studies in older adults are required to examine the potentially transient nature of BDNF and its relationship with resistance training.

Despite our reported findings, it is important that the results be interpreted within the context of this study. Although our intervention was designed to be 6 weeks in duration as a first attempt to explore and characterize neural performance changes, we may be able to gain further information on the trainability of specific aspects of cognition with an interventional study of a longer duration. Additionally, as a preliminary study to explore such changes, our results were interpreted using magnitude-based inferences to reduce errors in interpretation and to identify clinically relevant outcomes. Although confidence limits for clinical interpretations are derived from $p$ values obtained from independent statistical mean comparisons, clinical significance may not always align with statistical significance. Further, although our study was designed to first assess quantifiable outcomes on specified neural performance tests, future studies may yield greater insights into the mechanisms of the adaptations that occur. Finally, how such improvements translate to success in performing activities of daily living remains unknown, and this may provide a fruitful area for future research.

\section{Practical Applications}

There is a pressing need for effective interventions aimed at offsetting cognitive decline, especially because few pharmacological treatment options exist. Resistance exercise, in addition to the well-known physical benefits, may also provide clinically meaningful cognitive benefits in older adults. The results of this study support the use of resistance exercise as a means to potentially preserve or improve spatial awareness and visual and physical reaction times in older adults. Both spatial awareness and reaction are essential to 
accident avoidance in everyday living because they enable the individual to perceive and react to the surrounding environment. Thus, in accordance with the public health recommendations that older adults partake in resistance exercise training to improve physical health, further motivation to adhere to a structured and well-planned resistance exercise program should be encouraged by the cognitive benefits. Strength and conditioning professionals working with older adults should promote and encourage the potential cognitive benefits of resistance exercise to help clients attain a generally higher quality of life.

\section{ACKNOWLedGMents}

The authors wish to thank the dedicated group of research participants who volunteered their time, effort, and commitment to make this research possible. This research was conducted with research grants from the Learning Institute for Elders and the Toni Jennings Exceptional Education Institute.

\section{REFERENCES}

1. Aagaard, P, Suetta, C, Caserotti, P, Magnusson, SP, and Kjaer, M. Role of the nervous system in sarcopenia and muscle atrophy with aging: Strength training as a countermeasure. Scand J Med Sci Sports 20: 49-64, 2010

2. Barnes, DE, Yaffe, K, Satariano, WA, and Tager, IB. A longitudinal study of cardiorespiratory fitness and cognitive function in healthy older adults. J Am Geriatr Soc 51: 459-465, 2003.

3. Batterham, AM and Hopkins, WG. Making meaningful inferences about magnitudes. Sportscience 9: 6-13, 2005.

4. Bilimoria, PM and Bonni, A. Molecular control of axon branching. Neuroscientist 19: 16-24, 2013.

5. Bolstad, CA. Situation awareness: Does it change with age? Presented at Proceedings of the Human Factors and Ergonomics Society Annual Meeting, 2001.

6. Brummer, V, Schneider, S, Abel, T, Vogt, T, and Struder, HK. Brain cortical activity is influenced by exercise mode and intensity. Med Sci Sports Exerc 43: 1863-1872, 2011.

7. Bugg, JM and Head, D. Exercise moderates age-related atrophy of the medial temporal lobe. Neurobiol Aging 32: 506-514, 2011.

8. Cassilhas, RC, Lee, KS, Fernandes, J, Oliveira, MG, Tufik, S, Meeusen, R, and de Mello, MT. Spatial memory is improved by aerobic and resistance exercise through divergent molecular mechanisms. Neuroscience 202: 309-317, 2012.

9. Colcombe, $\mathrm{S}$ and Kramer, AF. Fitness effects on the cognitive function of older adults: A meta-analytic study. Psychol Sci 14: 125-130, 2003.

10. Davranche, K, Burle, B, Audiffren, M, and Hasbroucq, T. Physical exercise facilitates motor processes in simple reaction time performance: An electromyographic analysis. Neurosci Lett 396: 54-56, 2006.

11. Deschenes, MR. Motor unit and neuromuscular junction remodeling with aging. Curr Aging Sci 4: 209-220, 2011.

12. Diniz, BS and Teixeira, AL. Brain-derived neurotrophic factor and Alzheimer's disease: Physiopathology and beyond. Neuromolecular Med 13: 217-222, 2011.

13. Endsley, MR and Bolstad, CA. Individual differences in pilot situation awareness. Int J Aviat Psychol 4: 241-264, 1994.

14. Erickson, KI, Miller, DL, and Roecklein, KA. The aging hippocampus: Interactions between exercise, depression, and BDNF. Neuroscientist 18: 82-97, 2012.
15. Faubert, J and Sidebottom, L. Perceptual-cognitive training of athletes. J Clin Sport Psychol 6: 85-102, 2012.

16. Ferris, LT, Williams, JS, and Shen, CL. The effect of acute exercise on serum brain-derived neurotrophic factor levels and cognitive function. Med Sci Sports Exerc 39: 728-734, 2007.

17. Fiorentini, A, Porciatti, V, Morrone, MC, and Burr, DC. Visual ageing: Unspecific decline of the responses to luminance and colour. Vision Res 36: 3557-3566, 1996.

18. Galvin, JE, Powlishta, KK, Wilkins, K, McKeel, DW Jr, Xiong, C, Grant, E, Storandt, M, and Morris, JC. Predictors of preclinical Alzheimer disease and dementia: A clinicopathologic study. Arch Neurol 62: 758-765, 2005.

19. Gottsdanker, R. Age and simple reaction time. J Gerontol 37: 342348, 1982.

20. Hamilton, JM, Salmon, DP, Galasko, D, Raman, R, Emond, J, Hansen, LA, Masliah, E, and Thal, LJ. Visuospatial deficits predict rate of cognitive decline in autopsy-verified dementia with Lewy bodies. Neuropsychology 22: 729-737, 2008.

21. Hardy, SE and Studenski, SA. Fatigue and function over 3 years among older adults. J Gerontol A Biol Sci Med Sci 63: 1389-1392, 2008

22. Hopkins, WG. Linear models and effect magnitudes for research, clinical and practical applications. Sportscience 14: 49-57, 2010.

23. Hopkins, WG, Marshall, SW, Batterham, AM, and Hanin, J. Progressive statistics for studies in sports medicine and exercise science. Med Sci Sports Exerc 41: 3-13, 2009.

24. Karnath, HO, Ferber, S, and Himmelbach, M. Spatial awareness is a function of the temporal not the posterior parietal lobe. Nature 411: 950-953, 2001.

25. Klavora, P, Gaskovski, P, and Forsyth, RD. Test-retest reliability of three Dynavision tasks. Percept Mot Skills 80: 607-610, 1995.

26. Lambert, $\mathrm{CP}$ and Evans, WJ. Adaptations to aerobic and resistance exercise in the elderly. Rev Endocr Metab Disord 6: 137-143, 2005.

27. Lardon, MT and Polich, J. EEG changes from long-term physical exercise. Biol Psychol 44: 19-30, 1996.

28. Legault, I, Allard, R, and Faubert, J. Healthy older observers show equivalent perceptual-cognitive training benefits to young adults for multiple object tracking. Front Psychol 4: 323, 2013.

29. Lindvall, O, Kokaia, Z, Bengzon, J, Elmer, E, and Kokaia, M. Neurotrophins and brain insults. Trends Neurosci 17: 490-496, 1994.

30. Liu-Ambrose, T, Nagamatsu, LS, Voss, MW, Khan, KM, and Handy, TC. Resistance training and functional plasticity of the aging brain: A 12-month randomized controlled trial. Neurobiol Aging 33: 1690-1698, 2012.

31. Nagamatsu, LS, Handy, TC, Hsu, CL, Voss, M, and Liu-Ambrose, T. Resistance training promotes cognitive and functional brain plasticity in seniors with probable mild cognitive impairment. Arch Intern Med 172: 666-668, 2012.

32. Pannese, E. Morphological changes in nerve cells during normal aging. Brain Struct Funct 216: 85-89, 2011.

33. Park, HL, O'Connell, JE, and Thomson, RG. A systematic review of cognitive decline in the general elderly population. Int J Geriatr Psychiatry 18: 1121-1134, 2003.

34. Patten, C, Kamen, G, and Rowland, DM. Adaptations in maximal motor unit discharge rate to strength training in young and older adults. Muscle Nerve 24: 542-550, 2001.

35. Pirozzolo, FJ and Hansch, EC. Oculomotor reaction time in dementia reflects degree of cerebral dysfunction. Science 214: 349-351, 1981.

36. Porciatti, V, Fiorentini, A, Morrone, MC, and Burr, DC. The effects of ageing on reaction times to motion onset. Vision Res 39: 21572164, 1999.

37. Robertson, RJ, Goss, FL, Rutkowski, J, Lenz, B, Dixon, C, Timmer, J, Frazee, K, Dube, J, and Andreacci, J. Concurrent validation of the OMNI perceived exertion scale for resistance exercise. Med Sci Sports Exerc 35: 333-341, 2003. 
38. Sale, DG. Neural adaptation to resistance training. Med Sci Sports Exerc 20: S135-S145, 1988.

39. Salthouse, TA. Selective influences of age and speed on associative memory. Am J Psychol 108: 381-396, 1995.

40. Salthouse, TA and Meinz, EJ. Aging, inhibition, working memory, and speed. J Gerontol B Psychol Sci Soc Sci 50: P297-P306, 1995.

41. Schmidt-Kassow, M, Schadle, S, Otterbein, S, Thiel, C, Doehring, A, Lotsch, J, and Kaiser, J. Kinetics of serum brain-derived neurotrophic factor following low-intensity versus high-intensity exercise in men and women. Neuroreport 23: 889-893, 2012.

42. Spirduso, WW. Reaction and movement time as a function of age and physical activity level. J Gerontol 30: 435-440, 1975.

43. Surwillo, WW. The relation of decision time to brain wave frequency and to age. Electroencephalogr Clin Neurophysiol 16: 510514, 1964.

44. Swardfager, W, Herrmann, N, Marzolini, S, Saleem, M, Shammi, P, Oh, PI, Albert, PR, Daigle, M, Kiss, A, and Lanctot, KL. Brain derived neurotrophic factor, cardiopulmonary fitness and cognition in patients with coronary artery disease. Brain Behav Immun 25: 1264-1271, 2011.

45. Tapia-Arancibia, L, Aliaga, E, Silhol, M, and Arancibia, S. New insights into brain BDNF function in normal aging and Alzheimer disease. Brain Res Rev 59: 201-220, 2008.

46. van Gelder, BM, Tijhuis, MA, Kalmijn, S, Giampaoli, S, Nissinen, A, and Kromhout, D. Physical activity in relation to cognitive decline in elderly men: The FINE study. Neurology 63: 2316-2321, 2004.

47. Vaynman, SS, Ying, Z, Yin, D, and Gomez-Pinilla, F. Exercise differentially regulates synaptic proteins associated to the function of BDNF. Brain Res 1070: 124-130, 2006.

48. Weuve, J, Kang, JH, Manson, JE, Breteler, MM, Ware, JH, and Grodstein, F. Physical activity, including walking, and cognitive function in older women. JAMA 292: 1454-1461, 2004.

49. Wilson, PN, Foreman, N, and Stanton, D. Improving spatial awareness in physically disabled children using virtual environments. Presented at IEE Colloquium on Simulation in Medicine, 1998 